\title{
THE INVERSE HULL OF 0-LEFT CANCELLATIVE SEMIGROUPS
}

\author{
R. EXel AND B. Steinberg
}

\begin{abstract}
Given a semigroup $S$ with zero, which is left-cancellative in the sense that $s t=s r \neq 0$ implies that $t=r$, we construct an inverse semigroup called the inverse hull of $S$, denoted $\mathfrak{H}(S)$. When $S$ admits least common multiples, in a precise sense defined below, we study the idempotent semilattice of $\mathfrak{H}(S)$, with a focus on its spectrum. When $S$ arises as the language semigroup for a subsift $X$ on a finite alphabet, we discuss the relationship between $\mathfrak{H}(S)$ and several $\mathrm{C}^{*}$-algebras associated to $X$ appearing in the literature.
\end{abstract}

\section{Introduction.}

The goal of this note is to announce a series of results about semigroups, together with applications to $\mathrm{C}^{*}$ algebras, whose proofs will appear in later paper. The theory of semigroup $\mathrm{C}^{*}$-algebras has a long history, beginning with Coburn's work [4] and [5] where the $\mathrm{C}^{*}$-algebra of the additive semigroup of the natural numbers is studied in connection to Toeplitz operators. In [14] G. Murphy generalized this construction to the positive cone of an ordered group, and later to left-cancellative semigroups $([\mathbf{1 5}],[\mathbf{1 6}])$. The $\mathrm{C}^{*}$-algebras studied by Murphy turned out to be too wild, even for nice looking semigroups such as $\mathbb{N} \times \mathbb{N}$, and this prompted $\mathrm{Li}[\mathbf{1 0}]$ to introduce an alternative $\mathrm{C}^{*}$-algebra for a left-cancellative semigroup. By definition a semigroup $S$ is said to be left-cancellative provided, for every $r, s, t \in S$, one has that

$$
s t=s r \Rightarrow t=r \text {. }
$$

Many interesting semigroups in the literature possess a zero element, namely an element 0 such that

$$
s 0=0 s=0,
$$

for every $s$, and it is obvious that the presence of a zero prevents a semigroup from being left-cancellative. In this work we focus on 0-left-cancellative semigroups, meaning that (1.1) is required to hold only when the terms in its antecedent are supposed to be nonzero. This dramatically opens up the scope of applications including a wealth of interesting semigroups, such as those arising from subshifts and, more generaly, languages over a fixed alphabet. This also allows for the inclusion of categories and the semigroupoids of [7], once the multiplication is extended to all pairs of elements by setting undefined products to zero.

Starting with a 0-left-cancellative semigroup $S$, the crucial point is to first build an inverse semigroup $\mathfrak{H}(S)$, which we call the inverse hull of $S$, by analogy with $[\mathbf{3}],[\mathbf{2}]$, from where one may invoke any of the now standard constructions of $\mathrm{C}^{*}$-algebras from inverse semigroups, such as the tight $\mathrm{C}^{*}$-algebra $[\mathbf{7}]$ or Paterson's [18] universal $\mathrm{C}^{*}$-algebras. In fact this endeavor requires a lot more work regarding the passage from the original semigroup to its inverse hull, rather than the much better understood passage from there to the $\mathrm{C}^{*}$ algebras. Particularly demanding is the work geared towards understanding the idempotent semilattice of $\mathfrak{H}(S)$, which we denote by $\mathfrak{E}(S)$, as well as its spectrum. By a standard gadget $\mathfrak{E}(S)$ is put in correspondence with a subsemilattice of the power set of $S \backslash\{0\}$, whose members we call the constructible sets, by analogy with a similar concept relevant to Li's work in [10].

Central to the study of the spectrum of $\mathfrak{E}(S)$ is the notion of strings, which are motivated by the description of the unit space of graph groupoids in terms of paths in the graph.

Regarding the problem of understanding the spectrum of $\mathfrak{E}(S)$, we believe the present work represents only a modest beginning in a mammoth task lying ahead. This impression comes from situations in which similar spectra have been more or less understood, such as in [8] and in [6], illustrating the high degree of complexity one should expect.

It is only in our final section that we return to considering $\mathrm{C}^{*}$-algebras where we discuss, from the present perspective, how the Matsumoto and Carlsen-Matsumoto $\mathrm{C}^{*}$-algebras associated to a given subshift arise from the consideration of the inverse hull of the associated language semigroup. None of these correspond to the more well known tight or Paterson's universal $\mathrm{C}^{*}$-algebras, but we show that they instead arise from reductions of the Paterson groupoid to closed invariant subsets of its unit space which hitherto have not been identified. 


\section{Representations of semigroups.}

Let $S$ be a semigroup, namely a nonempty set equipped with an associative operation.

A zero element for $S$ is a (necessarily unique) element $0 \in S$, satisfying

$$
s 0=0 s=0, \quad \forall s \in S .
$$

In what follows we will fix a semigroup $S$ possessing a zero element.

2.1. Definition. Let $\Omega$ be any set. By a representation of $S$ on $\Omega$ we shall mean any map

$$
\pi: S \rightarrow \mathcal{I}(\Omega)
$$

where $\mathcal{I}(\Omega)$ is the symmetric inverse semigroup ${ }^{1}$ on $\Omega$, such that

(i) $\pi_{0}$ is the empty map on $\Omega$, and

(ii) $\pi_{s} \circ \pi_{t}=\pi_{s t}$, for all $s$ and $t$ in $S$.

Given a set $\Omega$, and any subset $X \subseteq \Omega$, let id $\operatorname{li}_{X}$ denote the identity function on $X$, so that $\operatorname{id}_{X}$ an element of $E(\mathcal{I}(\Omega))$, the idempotent semilattice of $\mathcal{I}(\Omega)$. One in fact has that

$$
E(\mathcal{I}(\Omega))=\left\{\operatorname{id}_{X}: X \subseteq \Omega\right\}
$$

so we may identify $E(\mathcal{I}(\Omega))$ with the meet semilattice $\mathcal{P}(\Omega)$ formed by all subsets of $\Omega$.

2.2. Definition. Given a representation $\pi$ of $S$, for every $s$ in $S$ we will denote the domain of $\pi_{s}$ by $F_{s}^{\pi}$, and the range of $\pi_{s}$ by $E_{s}^{\pi}$, so that $\pi_{s}$ is a bijective mapping

$$
\pi_{s}: F_{s}^{\pi} \rightarrow E_{s}^{\pi} .
$$

When

$$
\Omega=\left(\bigcup_{s \in S} F_{s}^{\pi}\right) \cup\left(\bigcup_{s \in S} E_{s}^{\pi}\right),
$$

we will say that $\pi$ is an essential representation. We will moreover let

$$
f_{s}^{\pi}:=\pi_{s}^{-1} \pi_{s}=\operatorname{id}_{E_{s}^{\pi}} \quad \text { and } \quad e_{s}^{\pi}:=\pi_{s} \pi_{s}^{-1}=\operatorname{id}_{F_{s}^{\pi}} .
$$

Let us fix, for the time being, a representation $\pi$ of $S$ on $\Omega$. Whenever there is only one representation in sight we will drop the superscripts in $F_{s}^{\pi}, E_{s}^{\pi}, f_{s}^{\pi}$, and $e_{s}^{\pi}$, and adopt the simplified notations $F_{s}, E_{s}, f_{s}$, and $e_{s}$.

The following may be proved easily.

2.3. Proposition. Given $s$ and $t$ in $S$, one has that

(i) $\pi_{s} e_{t}=e_{s t} \pi_{s}$, and

(ii) $f_{t} \pi_{s}=\pi_{s} f_{t s}$.

\subsection{Definition.}

(i) The inverse subsemigroup of $\mathcal{I}(\Omega)$ generated by the set $\left\{\pi_{s}: s \in S\right\}$ will be denoted by $\mathcal{I}(\Omega, \pi)$.

(ii) Given any $X \in \mathcal{P}(\Omega)$ such that $\operatorname{id}_{X}$ belongs to $E(\mathcal{I}(\Omega, \pi))$, we will say $X$ is a $\pi$-constructible subset.

(iii) The collection of all $\pi$-constructible subsets of $\Omega$ will be denoted by $\mathcal{P}(\Omega, \pi)$. In symbols

$$
\mathcal{P}(\Omega, \pi)=\left\{X \in \mathcal{P}(\Omega): \operatorname{id}_{X} \in E(\mathcal{I}(\Omega, \pi))\right\} .
$$

Observe that $E_{s}$ and $F_{s}$ are $\pi$-constructible sets. For the special case of $s=0$, we have $E_{s}=F_{s}=\emptyset$, so the empty set is $\pi$-constructible as well.

Since $\mathcal{P}(\Omega, \pi)$ corresponds to the idempotent semilattice of $\mathcal{I}(\Omega, \pi)$ by definition, it is clear that $\mathcal{P}(\Omega, \pi)$ is a semilattice, and in particular the intersection of two $\pi$-constructible sets is again $\pi$-constructible.

\footnotetext{
1 The symmetric inverse semigroup on a set $\Omega$ is the inverse semigroup formed by all partially defined bijections on $\Omega$.
} 


\section{Cancellative semigroups.}

3.1. Definition. Let $S$ be a semigroup containing a zero element. We will say that $S$ is 0-left-cancellative if, for every $r, s, t \in S$,

$$
s t=s r \neq 0 \Rightarrow t=r,
$$

and 0 -right-cancellative if

$$
t s=r s \neq 0 \Rightarrow t=r .
$$

If $S$ is both 0-left-cancellative and 0-right-cancellative, we will say that $S$ is 0 -cancellative.

- In what follows we will fix a 0-left-cancellative semigroup $S$. In a few occasions we will also assume that $S$ is 0-right-cancellative.

For any $s$ in $S$ we will let

$$
F_{s}=\{x \in S: s x \neq 0\},
$$

and

$$
E_{s}=\{y \in S: y=s x \neq 0 \text {, for some } x \in S\}
$$

Observe that the correspondence " $x \rightarrow s x$ " gives a map from $F_{s}$ onto $E_{s}$, which is one-to-one by virtue of 0-left-cancellativity.

3.2. Definition. For every $s$ in $S$ we will denote by $\theta_{s}$ the bijective mapping given by

$$
\theta_{s}: x \in F_{s} \mapsto s x \in E_{s} .
$$

Observing that 0 is neither in $F_{s}$, nor in $E_{s}$, we see that these are both subsets of

$$
S^{\prime}:=S \backslash\{0\},
$$

so we may view $\theta_{s}$ as a partially defined bijection on $S^{\prime}$, which is to say that $\theta_{s} \in \mathcal{I}\left(S^{\prime}\right)$. We should also notice that when $s=0$, both $F_{s}$ and $E_{s}$ are empty, so $\theta_{s}$ is the empty map.

3.4. Proposition. The correspondence

$$
s \in S \mapsto \theta_{s} \in \mathcal{I}\left(S^{\prime}\right)
$$

is a representation of $S$ on $S^{\prime}$, henceforth called the regular representation of $S$.

Regarding the notations introduced in (2.2) in relation to the regular representation, notice that

$$
F_{s}=F_{s}^{\theta}, \quad \text { and } \quad E_{s}=E_{s}^{\theta} .
$$

3.5. Definition. A semigroup $S$ is called right reductive if it acts faithfully on the left of itself, that is, $s x=t x$ for all $x \in S$ implies $s=t$.

Of course every unital semigroup is right reductive. If $S$ is a right reductive 0-left cancellative semigroup, then it embeds in $\mathcal{I}\left(S^{\prime}\right)$ via $s \mapsto \theta_{s}$.

Observe that if $S$ is 0-right-cancellative, then a single $x$ for which $s x=r x$, as long as this is nonzero, is enough to imply that $s=t$. So, in a sense, right reductivity is a weaker version of 0-right-cancellativity.

3.6. Definition. The inverse hull of $S$, henceforth denoted by $\mathfrak{H}(S)$, is the inverse subsemigroup of $\mathcal{I}\left(S^{\prime}\right)$ generated by the set $\left\{\theta_{s}: s \in S\right\}$. Thus, in the terminology of (2.4.i) we have

$$
\mathfrak{H}(S)=\mathcal{I}\left(S^{\prime}, \theta\right) .
$$

The reader should compare the above with the notion of inverse hull defined in $[\mathbf{3}],[\mathbf{2}]$.

The collection of $\theta$-constructible subsets of $S^{\prime}$ is of special importance to us, so we would like to give it a special notation: 
3.7. Definition. The idempotent semilattice of $\mathfrak{H}(S)$, which we will tacitly identify with the semilattice of $\theta$-constructible subsets of $S^{\prime}$, will be denoted by $\mathfrak{E}(S)$. Thus, in the terminology of (2.4.iii) we have

$$
\mathfrak{E}(S)=\mathcal{P}\left(S^{\prime}, \theta\right) .
$$

It will be important to identify some properties of 0-left cancellative semigroups which will play a role later.

3.8. Proposition. Let $S$ be a 0-left cancellative semigroup.

(i) If $e$ is an idempotent element in $S$ and $s \in S \backslash\{0\}$, then es $\neq 0$ if and only if es $=s$, that is, $s \in e S \backslash\{0\}$.

(ii) If $s \in S \backslash\{0\}$, then $s \in s S$ if and only if $s e=s$ for a necessarily unique idempotent $e$.

(iii) If $s S=S$ and $S$ is right reductive, then $S$ is unital and $s$ is invertible.

A semigroup $S$ is said to have right local units if $S=S E(S)$, that is, for all $s \in S$, there exists an idempotent element $e$ in $S$ with $s e=s$. A unital semigroup has right local units for trivial reasons. If $S$ has right local units, then $s S=0$ implies that $s=0$. From Proposition (3.8) we obtain the following:

3.9. Corollary. Let $S$ be a 0-left cancellative semigroup. Then $S$ has right local units if and only if $s \in s S$ for all $s \in S$.

In a right reductive 0-left cancellative semigroup, the idempotents are orthogonal to each other:

3.10. Proposition. Let $S$ be right reductive 0-left cancellative semigroup and suppose that $e \neq f$ are distinct idempotents of $S$. Then ef $=0$.

If $S$ is a 0-left cancellative, right reductive semigroup with right local units, then for $s \in S \backslash\{0\}$, we denote by $s^{+}$the unique idempotent with $s s^{+}=s$. If $S$ is unital, then $s^{+}=1$. If $C$ is a left cancellative category, we can associate a semigroup $S(C)$ by letting $S(C)$ consist of the arrows of $C$ together with a zero element 0. Products that are undefined in $C$ are made zero in $S(C)$. It is straightforward to check that $S(C)$ is 0-left cancellative, right reducitve and has right local units. If $f: c \rightarrow d$ is an arrow of $C$, then $f^{+}=1_{c}$.

\section{Examples.}

If $G$ is any group, and $P$ is a subsemigroup of $G$, let $S=P \cup\{0\}$, where 0 is any element not belonging to $P$, with the obvious multiplication operation. Then $S$ is clearly a 0 -cancellative semigroup.

A more elaborate example is as follows: let $\Lambda$ be any finite or infinite set, henceforth called the alphabet, and let $\Lambda^{+}$be the free semigroup generated by $\Lambda$, namely the set of all finite words in $\Lambda$ of positive length (and hence excluding the empty word), equipped with the multiplication operation given by concatenation.

Let $L$ be a language on $\Lambda$, namely any nonempty subset of $\Lambda^{+}$. We will furthermore assume that $L$ is closed under prefixes and suffixes in the sense that, for every $\alpha$ and $\beta$ in $\Lambda^{+}$, one has

$$
\alpha \beta \in L \Rightarrow \alpha \in L \text {, and } \beta \in L .
$$

Define a multiplication operation on

$$
S:=L \cup\{0\},
$$

where 0 is any element not belonging to $L$, by

$$
\alpha \cdot \beta=\left\{\begin{array}{cl}
\alpha \beta, & \text { if } \alpha, \beta \neq 0, \text { and } \alpha \beta \in L, \\
0, & \text { otherwise. }
\end{array}\right.
$$

One may then prove that $S$ is a 0 -cancellative semigroup.

One important special case of the above example is based on subshifts. Given an alphabet $\Lambda$, as above, let $X \subseteq \Lambda^{\mathbb{N}}$ be any nonempty subset invariant under the left shift, namely the mapping $\sigma: \Lambda^{\mathbb{N}} \rightarrow \Lambda^{\mathbb{N}}$ given by

$$
\sigma\left(x_{1} x_{2} x_{3} \ldots\right)=x_{2} x_{3} x_{4} \ldots
$$


Let $L \subseteq \Lambda^{+}$be the language of $X$, namely the set of all finite words occuring in some infinite word belonging to $X$. Then $L$ is clearly closed under prefixes and suffixes, and hence we are back in the conditions of the above example.

The fact that $X$ is invariant under the left shift is indeed superfluous, as any nonempty subset $X \subseteq \Lambda^{\mathbb{N}}$ would lead to the same conclusion. However, languages arising from subshifts have been intensively studied in the literature, hence the motivation for the above example. The semigroup associated to the language of a shift was first studied in the early days of symbolic dynamics by Morse and Hedlund [12].

4.2. Definition. ([13]) We will say that a semigroup $S$ with zero is categorical at zero if, for every $r, s, t \in S$, one has that

$$
r s \neq 0 \text {, and } s t \neq 0 \Rightarrow r s t \neq 0 .
$$

The semigroup associated to a category is categorical at zero, whence the name. We may use the above ideas to produce a semigroup which is not categorical at zero: take any nonempty alphabet $\Lambda$, let $L$ be the language consisting of all words of length at most two, and let $S=L \cup\{0\}$, as described above. If $a, b$ and $c$, are members of $\Lambda$, we have that $a b c=0$, but $a b$ and $b c$ are nonzero, so $S$ is not categorical at zero.

In contrast, notice that if $S$ is the semigroup built as above from a Markov subshift, then $S$ is easily seen to be categorical at zero.

Another interesting example is obtained from self-similar graphs [9]. Let $G$ be a discrete group, $E=$ $\left(E^{0}, E^{1}\right)$ be a graph with no sources, $\sigma$ be an action of $G$ by graph automorphisms on $E$, and

$$
\varphi: G \times E^{1} \rightarrow G
$$

be a one-cocycle for the restriction of $\sigma$ to $E^{1}$, which moreover satisfies

$$
\sigma_{\varphi(g, e)}(x)=\sigma_{g}(x) \quad \forall g \in G \quad \forall e \in E^{1} \quad \forall x \in E^{0} .
$$

Let $E^{*}$ be the set of all finite paths ${ }^{2}$ on $E$, including the vertices, which are seen as paths of length zero, and put

$$
S=\left(E^{*} \times G\right) \cup\{0\} .
$$

Given nonzero elements $(\alpha, g)$ and $(\beta, h)$ in $S$, define

$$
(\alpha, g)(\beta, h)=\left\{\begin{array}{cl}
\left(\alpha \sigma_{g}(\beta), \varphi(g, \beta) h\right), & \text { if } s(\alpha)=r\left(\sigma_{g}(\beta)\right), \\
0, & \text { otherwise. }
\end{array}\right.
$$

One may then show that $S$ is a categorical at zero, 0-left-cancellative semigroup, which is 0-rightcancellative if and only if $(G, E)$ is pseudo-free in the sense of [9: Proposition 5.6].

\section{Least common multiples.}

We now wish to introduce a special class of semigroups, but for this we must first consider the question of divisibility.

5.1. Definition. Given $s$ and $t$ in a semigroup $S$, we will say that $s$ divides $t$, in symbols

$$
s \mid t
$$

or that $t$ is a multiple of $s$, when either $s=t$, or there is some $u$ in $S$ such that $s u=t$. In other words,

$$
t \in\{s\} \cup s S \text {. }
$$

We observe that division is a reflexive and transitive relation, so it may be seen as a (not necessarily anti-symmetric) order relation.

For the strict purpose of simplifying the description of the division relation, regardless of whether or not $S$ is unital, we shall sometimes employ the unitized semigroup

$$
\tilde{S}:=S \cup\{1\},
$$

where 1 is any element not belonging to $S$, made to act like a unit for $S$. For every $s$ and $t$ in $S$ we therefore have that

$$
s \mid t \Longleftrightarrow \exists u \in \tilde{S}, \text { su }=t .
$$

Having enlarged our semigroup, we might as well extend the notion of divisibility:

\footnotetext{
2 We adopt the functorial point of view so a path is a sequence $e_{1} \ldots e_{n}$ of edges, such that $s\left(e_{i}\right)=r\left(e_{i+1}\right)$, for all $i$.
} 
5.3. Definition. Given $v$ and $w$ in $\tilde{S}$, we will say that $v \mid w$ when there exists some $u$ in $\tilde{S}$, such that $v u=w$.

Notice that if $v$ and $w$ are in $S$, then the above notion of divisibility coincides with the previous one by (5.2). Analysing the new cases where this extended divisibility may or may not apply, notice that:

$$
\begin{aligned}
& \forall w \in \tilde{S}, \quad 1 \mid w, \\
& \forall v \in \tilde{S}, \quad v \mid 1 \Longleftrightarrow v=1 .
\end{aligned}
$$

The introduction of $\tilde{S}$ brings with it several pitfalls, not least because $\tilde{S}$ might not be 0-left-cancellative: when $S$ already has a unit, say $1_{S}$, then in the identity " $s 1_{S}=s 1$ ", we are not allowed to left-cancel $s$, since $1_{S} \neq 1$. One should therefore be very careful when working with $\tilde{S}$.

5.5. Definition. Let $S$ be a semigroup and let $s, t \in S$. We will say that an element $r \in S$ is a least common multiple for $s$ and $t$ when

(i) $s S \cap t S=r S$,

(ii) both $s$ and $t$ divide $r$.

Observe that when $S$ has right local units then $r \in r S$, by (3.9), and hence condition (5.5.i) trivially implies (5.5.ii), so the former condition alone suffices to define least common multiples. However in a unitless semigroup condition (5.5.i) may hold while (5.5.ii) fails. Nevertheless, when $s S \cap t S=\{0\}$, then 0 is a least common multiple for $s$ and $t$ because $s$ and $t$ always divide 0 .

Regardless of the existence of right local units, notice that condition (5.5.ii) holds if and only if $r \tilde{S} \subseteq$ $s \tilde{S} \cap t \tilde{S}$, and therefore one has that $r$ is a least common multiple for $s$ and $t$ if and only if

$$
s S \cap t S=r S \subseteq r \tilde{S} \subseteq s \tilde{S} \cap t \tilde{S} .
$$

One may of course think of alernative definitions for the concept of least common multiples, fiddling with the above ideas in many different ways. However (5.5) seems to be the correct choice, at least from the point of view of the theory we are about to develop.

5.7. Definition. We shall say that a semigroup $S$ admits least common multiples if there exists a least common multiple for each pair of elements of $S$.

The language semigroup of (4.1) is easily seen to be an example of a semigroup admitting least common multiples.

Another interesting example is obtained from the quasi-lattice ordered groups of $[\mathbf{1 7}]$, which we would now like to briefly describe.

Given a group $G$ and a unital subsemigroup $P \subseteq G$, one defines a partial order on $G$ via

$$
x \leq y \Longleftrightarrow x^{-1} y \in P .
$$

The quasi-lattice condition says that, whenever elements $x$ and $y$ in $G$ admit a common upper bound, namely an element $z$ in $G$ such that $z \geq x$ and $z \geq y$, then there exists a least common upper bound, usually denoted $x \vee y$.

Under this situation, consider the semigroup $S=P \cup\{0\}$, obtained by adjoining a zero to $P$. Then, for every nonzero $s$ in $S$, i.e. for $s$ in $P$, one has that

$$
s S=\{x \in P: x \geq s\} \cup\{0\},
$$

so that the multiples of $s$ are precisely the upper bounds of $s$ in $P$, including zero.

If $t$ is another nonzero element in $S$, one therefore has that $s$ and $t$ admit a nonzero commun multiple if and only if $s$ and $t$ admit a common upper bound in $P$, in which case $s \vee t$ is a least common multiple of $s$ and $t$.

On the other hand, when $s$ and $t$ admit no common upper bound, then obviously $s \vee t$ does not exist, but still $s$ and $t$ admit a least common multiple in $S$, namely 0 .

Summarizing we have the following: 
5.8. Proposition. Let $(G, P)$ be a quasi-lattice ordered group. Then the semigroup $S:=P \cup\{0\}$ admits least common multiples.

- From this point on we will fix a 0-left-cancellative, semigroup $S$ admitting least common multiples.

5.9. Proposition. Given $u$ and $v$ in $\tilde{S}$, there exists $w$ in $\tilde{S}$ such that

(i) $u S \cap v S=w S$,

(ii) both $u$ and $v$ divide $w$.

Proof. When $u$ and $v$ lie in $S$, it is enough to take $w$ to be a (usual) least common multiple of $u$ and $v$. On the other hand, if $u=1$, one takes $w=v$, and if $v=1$, one takes $w=u$.

Based on the above we may extend the notion of least common multiples to $\tilde{S}$, as follows:

5.10. Definition. Given $u$ and $v$ in $\tilde{S}$, we will say that an element $w$ in $\tilde{S}$ is a least common multiple of $u$ and $v$, provided (5.9.i-ii) hold. In the exceptional case that $u=v=1$, only $w=1$ will be considered to be a least common multiple of $u$ and $v$, even though there might be another $w$ in $S$ satisfying (5.9.i-ii).

It is perhaps interesting to describe the exceptional situation above, where we are arbitrarily prohibiting by hand that an element of $S$ be considered as a least common multiple of 1 and itself, even though it would otherwise satisfy all of the required properties. If $w \in S$ is such an element, then

$$
w S=1 S \cap 1 S=S,
$$

so, in case we also assume that $S$ is right-reductive, we deduce from (3.8.iii) that $S$ is unital and $w$ is invertible. Thus, in hindsight it might not have been such a good idea to add an external unit to $S$ after all.

On the other hand, when $s$ and $t$ lie in $S$, it is not hard to see that any least common multiple of $s$ and $t$ in the new sense of (5.10) must belong to $S$, and hence it must also be a least common multiple in the old sense of (5.5).

Given a representation $\pi$ of $S$ on a set $\Omega$, we will now concentrate our attention on giving a concrete description for the inverse semigroup $\mathcal{I}(\Omega, \pi)$ defined in (2.4), provided $\pi$ satisfies certain special properties, which we will now describe.

Initially notice that if $s \mid r$, then the range of $\pi_{r}$ is contained in the range of $\pi_{s}$ because either $r=s$, or $r=s u$, for some $u$ in $S$, in which case $\pi_{r}=\pi_{s} \pi_{u}$. So, using the notation introduced in (2.2),

$$
E_{r}^{\pi} \subseteq E_{s}^{\pi}
$$

When $r$ is a common multiple of $s$ and $t$, it then follows that

$$
E_{r}^{\pi} \subseteq E_{s}^{\pi} \cap E_{t}^{\pi}
$$

5.11. Definition. A representation $\pi$ of $S$ is said to respect least common multiples if, whenever $r$ is a least common multiple of elements $s$ and $t$ in $S$, one has that $E_{r}^{\pi}=E_{s}^{\pi} \cap E_{t}^{\pi}$.

As an example, notice that the regular representation of $S$, defined in (3.4), satisfies the above condition since the fact that $r S=s S \cap t S$ implies that

$$
E_{r}^{\theta}=r S \backslash\{0\}=(s S \cap t S) \backslash\{0\}=(s S \backslash\{0\}) \cap(t S \backslash\{0\})=E_{s}^{\theta} \cap E_{t}^{\theta} .
$$

- From now on we will fix a representation $\pi$ of $S$ on a set $\Omega$, assumed to respect least common multiples. Since $\pi$ will be the only representation considered for a while, we will use the simplified notations $F_{s}, E_{s}$, $f_{s}$, and $e_{s}$.

There is a cannonical way to extend $\pi$ to $\tilde{S}$ by setting

$$
F_{1}=E_{1}=\Omega, \quad \text { and } \quad \pi_{1}=\operatorname{id}_{\Omega} .
$$

It is evident that $\pi$ remains a multiplicative map after this extension. Whenever we find it convenient we will therefore think of $\pi$ as defined on $\tilde{S}$ as above. We will accordingly extend the notations $f_{s}$ and $e_{s}$ to allow for any $s$ in $\tilde{S}$, in the obvious way. 
5.13. Proposition. Let $\pi$ be a representation of $S$ on a set $\Omega$. If $\pi$ respects least common multiples then so does its natural extension to $\tilde{S}$. Precisely, if $u$ and $v$ are elements of $\tilde{S}$, and if $w \in \tilde{S}$ is a least common multiple of $u$ and $v$, then $E_{w}=E_{u} \cap E_{v}$.

5.14. Definition. Given a representation $\pi$ of $S$, and given any nonempty finite subset $\Lambda \subseteq \tilde{S}$, we will let

$$
F_{\Lambda}^{\pi}=\bigcap_{u \in \Lambda} F_{u}^{\pi}, \quad \text { and } \quad f_{\Lambda}^{\pi}=\prod_{u \in \Lambda} f_{u}^{\pi}
$$

When there is only one representation of $S$ in sight, as in the present moment, we will drop the superscripts and use the simplified notations $F_{\Lambda}$ and $f_{\Lambda}$.

We should remark that, since each $f_{s}$ is the identity map on $F_{s}$, one has that $f_{\Lambda}$ is the identity map on $F_{\Lambda}$.

Also notice that, since $f_{1}=\mathrm{id}_{\Omega}$, the presence of 1 in $\Lambda$ has no effect in the sense that $f_{\Lambda}=f_{\Lambda \cup\{1\}}$, for every $\Lambda$. Thus, whenever convenient we may assume that $1 \in \Lambda$.

As already indicated we are interested in obtaining a description for the inverse semigroup $\mathcal{I}(\Omega, \pi)$. In that respect it is interesting to observe that most elements of the form $f_{\Lambda}$ belong to $\mathcal{I}(\Omega, \pi)$, but there is one exception, namely when $\Lambda=\{1\}$. In this case we have

$$
f_{\{1\}}=\operatorname{id}_{\Omega},
$$

which may or may not lie in $\mathcal{I}(\Omega, \pi)$. However, when $\Lambda \cap S \neq \emptyset$, then surely

$$
f_{\Lambda} \in \mathcal{I}(\Omega, \pi) .
$$

We should furthermore remark that, whenever we are looking at a term of the form $\pi_{u} f_{\Lambda} \pi_{v}^{-1}$, we may assume that $u, v \in \Lambda$, because

$$
\begin{aligned}
& \pi_{u} f_{\Lambda} \pi_{v}^{-1}=\pi_{u} \pi_{u}^{-1} \pi_{u} f_{\Lambda} \pi_{v}^{-1} \pi_{v} \pi_{v}^{-1}= \\
& =\pi_{u} f_{u} f_{\Lambda} f_{v} \pi_{v}^{-1}=\pi_{u} f_{\{u\} \cup \Lambda \cup\{v\}} \pi_{v}^{-1},
\end{aligned}
$$

so $\Lambda$ may be replaced by $\{u\} \cup \Lambda \cup\{v\}$ without altering the above term. Moreover, as in (5.15), observe that when $\Lambda \cap S \neq \emptyset$, then

$$
\pi_{u} f_{\Lambda} \pi_{v}^{-1} \in \mathcal{I}(\Omega, \pi) .
$$

5.17. Theorem. Let $S$ be a 0-left-cancellative semigroup admitting least common multiples. Also let $\pi$ be a representation of $S$ on a set $\Omega$, assumed to respect least common multiples. Then the generated inverse semigroup $\mathcal{I}(\Omega, \pi)$ is given by

$$
\mathcal{I}(\Omega, \pi)=\left\{\pi_{u} f_{\Lambda} \pi_{v}^{-1}: \Lambda \subseteq \tilde{S} \text { is finite, } \Lambda \cap S \neq \emptyset \text {, and } u, v \in \Lambda\right\} .
$$

With this we may describe the constructible sets in a very concrete way.

5.18. Proposition. Under the assumptions of (5.17), the $\pi$-constructible subsets of $\Omega$ are precisely the sets of the form

$$
X=\pi_{u}\left(F_{\Lambda}\right),
$$

where $\Lambda \subseteq \tilde{S}$ is a finite subset, $\Lambda \cap S \neq \emptyset$, and $u \in \Lambda$.

Recalling that the regular representation of $S$ respects least common multiples, our last two results apply to give:

5.19. Corollary. Let $S$ be a 0-left-cancellative semigroup admitting least common multiples. Then

$$
\mathfrak{H}(S)=\left\{\theta_{u} f_{\Lambda} \theta_{v}^{-1}: \Lambda \subseteq \tilde{S} \text { is finite, } \Lambda \cap S \neq \emptyset, \text { and } u, v \in \Lambda\right\},
$$

and

$$
\mathfrak{E}(S)=\left\{u F_{\Lambda}, \Lambda \subseteq \tilde{S} \text { is finite, } \Lambda \cap S \neq \emptyset \text {, and } u \in \Lambda\right\} .
$$

If $S$ is categorical at zero and has right local units, then a more precise normal form can be obtained. 


\section{Strings.}

This section is intended to introduce a device which will be highly useful in the study of the spectrum of $\mathfrak{E}(S)$.

- Throughout this section $S$ will be a fixed 0-left-cancellative semigroup.

6.1. Definition. A nonempty subset $\sigma \subseteq S$ is said to be a string in $S$, if

(i) $0 \notin \sigma$,

(ii) for every $s$ and $t$ in $S$, if $s \mid t$, and $t \in \sigma$, then $s \in \sigma$,

(iii) for every $s_{1}$ and $s_{2}$ in $\sigma$, there is some $s$ in $\sigma$ such that $s_{1} \mid s$, and $s_{2} \mid s$.

An elementary example of a string is the set of divisors of any nonzero element $s$ in $S$, namely,

$$
\delta_{s}=\{t \in S: t \mid s\}
$$

Strings often contain many elements, but there are some exceptional strings consisting of a single semigroup element. To better study these it is useful to introduce some terminology.

6.3. Definition. Given $s$ in $S$ we will say that $s$ is:

(i) prime, if the only divisor of $s$ is $s$, itself, or, equivalently, if $\delta_{s}=\{s\}$,

(ii) irreducible, if there are no two elements $x$ and $y$ in $S$ such that $s=x y$, or, equivalently, if $s \notin S^{2}$.

It is evident that any irreducible element is prime, but there might be prime elements which are not irreducible. For example, in the semigroup $S=\{0, s, e\}$, with multiplication table given by

\begin{tabular}{|c|c|c|c|}
\hline$\times$ & 0 & $e$ & $s$ \\
\hline 0 & 0 & 0 & 0 \\
\hline$e$ & 0 & $e$ & 0 \\
\hline$s$ & 0 & $s$ & 0 \\
\hline
\end{tabular}

one has that $s$ is prime but not irreducible because $s=s e \in S^{2}$.

6.4. Proposition. A singleton $\{s\}$ is a string if and only if $s$ is prime.

Proof. If $s$ is prime then the singleton $\{s\}$ coincides with $\delta_{s}$, and hence it is a string. Conversely, supposing that $\{s\}$ is a string, we have by (6.1.ii) that $\delta_{s} \subseteq\{s\}$, from where it follows that $s$ is prime.

6.5. Definition. The set of all strings in $S$ will be denoted by $S^{\star}$.

From now on our goal will be to define an action of $S$ on $S^{\star}$.

6.6. Proposition. Let $\sigma$ be a string in $S$, and let $r \in S$. Then

(i) if 0 is not in $r \sigma$, one has that

$$
r * \sigma:=\{t \in S: t \mid r s, \text { for some } s \in \sigma\}
$$

is a string whose intersection with $r S$ is nonempty.

(ii) If $\sigma$ is a string whose intersection with $r S$ is nonempty, then

$$
r^{-1} * \sigma:=\{t \in S: r t \in \sigma\}
$$

is a string, and 0 is not in $r\left(r^{-1} * \sigma\right)$. 
It should be noted that, under the assumptions of (6.6.i), one has that

$$
r \sigma \subseteq r * \sigma,
$$

and in fact $r * \sigma$ is the hereditary closure of $r \sigma$ relative to the order relation given by division.

We may then define a representation of $S$ on the set $S^{\star}$ of all strings in $S$, as follows:

6.8. Proposition. For each $r$ in $S$, put

$$
F_{r}^{\star}=\left\{\sigma \in S^{\star}: r \sigma \not \supset 0\right\}, \quad \text { and } \quad E_{r}^{\star}=\left\{\sigma \in S^{\star}: \sigma \cap r S \neq \emptyset\right\} .
$$

Also let

$$
\theta_{r}^{\star}: F_{r}^{\star} \rightarrow E_{r}^{\star}
$$

be defined by $\theta_{r}^{\star}(\sigma)=r * \sigma$, for every $\sigma \in F_{r}^{\star}$. Then:

(i) $\theta_{r}^{\star}$ is bijective, and its inverse is the mapping defined by

$$
\sigma \in E_{r}^{\star} \mapsto r^{-1} * \sigma \in F_{r}^{\star} .
$$

(ii) Viewing $\theta^{\star}$ as a map from $S$ to $\mathcal{I}\left(S^{\star}\right)$, one has that $\theta^{\star}$ is a representation of $S$ on $S^{\star}$.

Useful alternative characterizations of $F_{r}^{\star}$ and $E_{r}^{\star}$ are as follows:

6.9. Proposition. Given $r$ in $S$, and given any string $\sigma$ in $S^{\star}$, one has that:

(i) $\sigma \in F_{r}^{\star} \Leftrightarrow \sigma \subseteq F_{r}^{\theta}$,

(ii) $\sigma \in E_{r}^{\star} \Leftrightarrow \sigma \cap E_{r}^{\theta} \neq \emptyset$,

(iii) $\sigma \in E_{r}^{\star} \Rightarrow r \in \sigma$. In addition, the converse holds provided $r \in r S$ (e.g. if $S$ has right local units).

Recall from (5.19) that, when $S$ has least common multiples, every $\theta$-constructible subset of $S^{\prime}$ has the form $\theta_{u}\left(F_{\Lambda}^{\theta}\right)$, where $\Lambda \subseteq \tilde{S}$ is finite, $\Lambda \cap S \neq \emptyset$, and $u \in \Lambda$. By analogy this suggests that it might also be useful to have a characterization of $\theta_{u}^{\star}\left(F_{\Lambda}^{\star}\right)$ along the lines of (6.9).

6.10. Proposition. Let $\Lambda$ be a finite subset of $\tilde{S}$ having a nonempty intersection with $S$, and let $u \in \Lambda$. Then $\theta_{u}^{\star}\left(F_{\Lambda}^{\star}\right)$ consists precisely of the strings $\sigma$ such that

$$
\emptyset \neq \sigma \cap E_{u}^{\theta} \subseteq \theta_{u}\left(F_{\Lambda}^{\theta}\right) .
$$

After (6.8) we now have two natural representations of $S$, namely the regular representation $\theta$ acting on $S^{\prime}$, and $\theta^{\star}$ acting on $S^{\star}$.

6.11. Proposition. The map

$$
\delta: s \in S^{\prime} \mapsto \delta_{s} \in S^{\star},
$$

where $\delta_{s}$ is defined in (6.2), is covariant relative to $\theta$ and $\theta^{\star}$.

Observe that the union of an increasing family of strings is a string, so any string is contained in a maximal one by Zorn's Lemma.

6.12. Definition. The subset of $S^{\star}$ formed by all maximal strings will be denoted by $S^{\infty}$.

Our next result says that $S^{\infty}$ is invariant under $\theta^{\star}$.

6.13. Proposition. For every $r$ in $S$, and for every maximal string $\sigma$ in $F_{r}^{\star}$, one has that $\theta_{r}^{\star}(\sigma)$ is maximal.

Observe that the above result says that $S^{\infty}$ is invariant under each $\theta_{r}^{\star}$, but not necessarily under $\theta_{r}^{\star-1}$.

An example to show that $S^{\infty}$ may indeed be non invariant under $\theta_{r}^{\star-1}$ is as follows. Consider the language $L$ on the alphabet $\Sigma=\{a, b\}$ given by

$$
L=\{a, b, a a, b a\} .
$$

Then, $\sigma=\{b, b a\}$ is a maximal string, while $\theta_{b}^{\star-1}=\{a\}$ is not maximal. 
6.14. Definition. By a representation of a given inverse semigroup $\mathcal{S}$ on a set $\Omega$ we shall mean any map

$$
\rho: \mathcal{S} \rightarrow \mathcal{I}(\Omega)
$$

such that $\rho(0)$ is the empty map, and for every $s$ and $t$ in $\mathcal{S}$, one has that $\rho(s t)=\rho(s) \rho(t)$, and $\rho\left(s^{-1}\right)=$ $\rho(s)^{-1}$.

6.15. Proposition. Let $S$ be a 0-left-cancellative semigroup admitting least common multiples. Then there exists a unique representation $\rho$ of $\mathfrak{H}(S)$ on $S^{\star}$, such that the following diagram commutes.

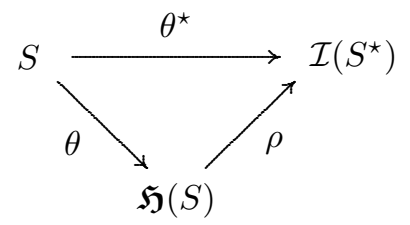

Observing that a homomorphism of inverse semigroups must restrict to the corresponding idempotent semilattices, we obtain the following:

6.16. Corollary. Let $S$ be a 0-left-cancellative semigroup admitting least common multiples. Then there exists a semilattice representation

$$
\varepsilon: \mathfrak{E}(S) \rightarrow \mathcal{P}\left(S^{\star}\right)
$$

such that

$$
\varepsilon\left(\theta_{u}\left(F_{\Lambda}^{\theta}\right)\right)=\theta_{u}^{\star}\left(F_{\Lambda}^{\star}\right)
$$

whenever $\Lambda$ is a finite subset of $\tilde{S}$ intersecting $S$ nontrivially, and $u \in \Lambda$.

Observing that $E_{r}^{\theta}=\theta_{r}\left(F_{r}^{\theta}\right)$, notice that

$$
\varepsilon\left(E_{r}^{\theta}\right)=\theta_{r}^{\star}\left(F_{r}^{\star}\right)=E_{r}^{\star} .
$$

\section{The spectrum of the semilattice of constructible sets.}

Let us now fix a 0-left-cancellative semigroup $S$ admitting least common multiples. It is our goal in this section to study the spectrum of $\mathfrak{E}(S)$.

Recall that if $\mathcal{E}$ is a semilattice with zero, the spectrum of $\mathcal{E}$ is the set of all semilattice homomorphisms $\varphi: \mathcal{E} \rightarrow\{0,1\}$, such that $\varphi(0)=0$. Here $\{0,1\}$ is equipped with its standard semilattice structure $0<1$.

Considering the representation

$$
\varepsilon: \mathfrak{E}(S) \rightarrow \mathcal{P}\left(S^{\star}\right)
$$

introduced in (6.16), and given $\sigma \in S^{\star}$, set

$$
\varphi_{\sigma}: X \in \mathfrak{E}(S) \mapsto[\sigma \in \varepsilon(X)] \in\{0,1\}
$$

It is clear that $\varphi_{\sigma}$ is a semilattice homomorphism, so it is a character as long as it is nonzero.

The question of whether or not $\varphi_{\sigma}$ is nonzero evidently boils down to the existence of some constructible set $X$ for which $\sigma \in \varepsilon(X)$. By (5.19) it is easy to see that for every constructible set $X$, there is some $r$ in $S$ such that $X \subseteq E_{r}$, or $X \subseteq F_{r}$. Therefore $\varphi_{\sigma}=0$ if and only if $\sigma$ is never in any $E_{r}^{\star}$ nor in any $F_{r}^{\star}$, that is,

$$
\sigma \nsubseteq F_{r}^{\theta}, \quad \text { and } \quad \sigma \cap E_{r}^{\theta}=\emptyset
$$

for every $r$ in $S$, by (6.9).

The second condition above implies that every element in $\sigma$ is irreducible, so it necessarily follows that $\sigma$ is a singleton, say $\sigma=\{s\}$, where $s$ is irreducible. In turn, the first condition above implies that $s$ lies in no $F_{r}^{\theta}$, whence $S s=0$. 


\subsection{Definition.}

(i) An element $s$ in $S$ will be called degenerate if $s$ is irreducible and $S s=\{0\}$.

(ii) A string $\sigma$ will be called degenerate if $\sigma=\{s\}$, where $s$ is a degenerate element.

(iii) The set of all non-degenerate strings will be denoted by $S_{\sharp}^{\star}$.

(iv) For every non-degenerate string $\sigma$, we shall denote by $\varphi_{\sigma}$ the character of $\mathfrak{E}(S)$ given by

$$
\varphi_{\sigma}(X)=[\sigma \in \varepsilon(X)], \quad \forall X \in \mathfrak{E}(S) .
$$

Suppose we are given $\varphi_{\sigma}$ and we want to recover $\sigma$ from $\varphi_{\sigma}$. In the special case in which $S$ has right local units, we have that

$$
\varphi_{\sigma}\left(E_{s}^{\theta}\right)=1 \Longleftrightarrow \sigma \in \varepsilon\left(E_{s}^{\theta}\right)=E_{s}^{\star} \stackrel{(6.9 . \text { iii })}{\Longleftrightarrow} s \in \sigma,
$$

so $\sigma$ is recovered as the set $\left\{s \in S: \varphi_{\sigma}\left(E_{s}^{\theta}\right)=1\right\}$. Without assuming right local units, the last part of (7.2) cannot be trusted, but it may be replaced with

$$
\cdots \stackrel{(6.9 . \mathrm{ii})}{\Longleftrightarrow} \sigma \cap E_{s}^{\theta} \neq \emptyset
$$

so we at least know which $E_{s}^{\theta}$ have a nonempty intersection with $\sigma$.

7.4. Proposition. Given any string $\sigma$, let the interior of $\sigma$ be defined by

$$
\stackrel{\circ}{\sigma}:=\{s \in S: \exists x \in S, s x \in \sigma\} .
$$

Then

$$
\stackrel{\circ}{\sigma}=\left\{s \in S: \varphi_{\sigma}\left(E_{s}^{\theta}\right)=1\right\} \text {. }
$$

Given any character $\varphi$ of $\mathfrak{E}(S)$, regardless of whether or not it is of the form $\varphi_{\sigma}$ as above, we may still consider the set

$$
\sigma_{\varphi}:=\left\{s \in S: \varphi\left(E_{s}^{\theta}\right)=1\right\},
$$

so that, when $\varphi=\varphi_{\sigma}$, we get $\sigma_{\varphi}=\stackrel{\circ}{\sigma}$.

7.6. Proposition. If $\varphi$ is any character of $\mathfrak{E}(S)$, and $\sigma_{\varphi}$ is nonempty, then $\sigma_{\varphi}$ is a string closed under least common multiples.

Based on (7.1.iv) we may define a map from the set of all non-degenerate strings to $\widehat{\mathfrak{E}}(S)$, the spectrum of $\mathfrak{E}(S)$, by

$$
\Phi: \sigma \in S_{\sharp}^{\star} \mapsto \varphi_{\sigma} \in \widehat{\mathfrak{E}}(S),
$$

but if we want the dual correspondence suggested by (7.5), namely

$$
\varphi \mapsto \sigma_{\varphi}
$$

to give a well defined map from $\widehat{\mathfrak{E}}(S)$ to $S^{\star}$, we need to worry about its domain because we have not checked that $\sigma_{\varphi}$ is always nonempty, and hence $\sigma_{\varphi}$ may fail to be a string. The appropriate domain is evidently given by the set of all characters $\varphi$ such that $\sigma_{\varphi}$ is nonempty but, before we formalize this map, it is interesting to introduce a relevant subsemilattice of $\mathfrak{E}(S)$.

7.9. Proposition. The subset of $\mathfrak{E}(S)$ given $b y^{3}$

$$
\mathfrak{E}_{1}(S)=\left\{s F_{\Lambda}^{\theta}, \Lambda \subseteq S \text { is finite, and } s \in \Lambda\right\},
$$

is an ideal of $\mathfrak{E}(S)$. Moreover, for every $X$ in $\mathfrak{E}(S)$, one has that $X$ lies in $\mathfrak{E}_{1}(S)$ if and only if $X \subseteq E_{s}^{\theta}$, for some $s$ in $S$. $S$.

3 This should be contrasted with (5.19), where the general form of an element of $\mathfrak{E}(S)$ is $u F_{\Lambda}^{\theta}$, where $u$ is in $\tilde{S}$, rather than 
Whenever $J$ is an ideal in a semilattice $\mathcal{E}$, there is a standard inclusion

$$
\varphi \in \hat{J} \mapsto \tilde{\varphi} \in \hat{E}
$$

where, for every $x$ in $E$, one has that $\tilde{\varphi}(x)=1$, if and only if there exists some $y$ in $J$ with $y \leq x$, and $\varphi(y)=1$. The next result is intended to distinguish the elements of the copy of $\widehat{\mathfrak{E}}_{1}(S)$ within $\widehat{\mathfrak{E}}(S)$ given by the above correspondence.

7.10. Proposition. Let $\varphi$ be a character on $\mathfrak{E}(S)$. Then the following are equivalent:

(i) $\varphi \in \widehat{\mathfrak{E}}_{1}(S)$,

(ii) $\varphi\left(E_{s}^{\theta}\right)=1$, for some $s$ in $S$,

(iii) $\sigma_{\varphi}$ is nonempty, and hence it is a string by (7.6).

If $S$ admits right local units, then $\mathfrak{E}_{1}(S)=\mathfrak{E}(S)$, so $\sigma_{\varphi}$ is a string for every character $\varphi \in \widehat{\mathfrak{E}}(S)$.

The vast majority of non-degenerate strings $\sigma$ lead to a character $\varphi_{\sigma}$ belonging to $\widehat{\mathfrak{E}}_{1}(S)$, but there are exceptions.

7.11. Proposition. If $\sigma$ is a non-degenerate string in $S_{\sharp}^{\star}$ then $\varphi_{\sigma}$ does not belong to $\widehat{\mathfrak{E}}_{1}(S)$ if and only if $\sigma=\{s\}$, where $s$ is an irreducible element of $S$.

By (7.10) we have that the largest set of characters on which the correspondence described in (7.8) produces a bona fide string is precisely $\widehat{\mathfrak{E}}_{1}(S)$, so we may now formaly introduce the map suggested by that correspondence.

7.12. Definition. We shall let

$$
\Sigma: \widehat{\mathfrak{E}}_{1}(S) \mapsto S^{\star},
$$

be the map given by

$$
\Sigma(\varphi)=\sigma_{\varphi}=\left\{s \in S: \varphi\left(E_{s}^{\theta}\right)=1\right\}, \quad \forall \varphi \in \widehat{\mathfrak{E}}_{1}(S) .
$$

For every string $\sigma$, excluding the exceptional ones discussed in (7.11), we then have that

$$
\Phi(\sigma)=\varphi_{\sigma} \in \widehat{\mathfrak{E}}_{1}(S),
$$

and

$$
\Sigma(\Phi(\sigma))=\stackrel{\circ}{\sigma}
$$

by $(7.4)$.

7.14. Definition. A string $\sigma$ in $S$ will be termed open if $\sigma=\stackrel{\circ}{\sigma}$.

The nicest situation is for open strings:

7.15. Proposition. If $\sigma$ is an open string, then

(i) $\sigma$ is non-degenerate,

(ii) $\Phi(\sigma) \in \widehat{\mathfrak{E}}_{1}(S)$, and

(iii) $\Sigma(\Phi(\sigma))=\sigma$.

Given that the composition $\Sigma \circ \Phi$ is so well behaved for open strings, we will now study the reverse composition $\Phi \circ \Sigma$ on a set of characters related to open strings.

7.16. Definition. A character $\varphi$ in $\widehat{\mathfrak{E}}(S)$ will be called an open character if $\sigma_{\varphi}$ is a (nonempty) open string.

We remark that every open character belongs to $\widehat{\mathfrak{E}}_{1}(S)$ by $(7.10)$, although not all characters in $\widehat{\mathfrak{E}}_{1}(S)$ are open.

By (7.15) it is clear that $\varphi_{\sigma}$ is an open character for every open string $\sigma$.

If $S$ admits right local units, we have seen that every string in $S^{\star}$ is open, and also that $\sigma_{\varphi}$ is a string for every character. Therefore every character in $\widehat{\mathfrak{E}}(S)$ is open.

The composition $\Phi \circ \Sigma$ is not as well behaved as the one discussed in (7.15), but there is at least some relationship between a character $\varphi$ and its image under $\Phi \circ \Sigma$, as we shall now see. 
7.17. Proposition. Given any open character $\varphi$, one has that

$$
\varphi \leq \Phi(\Sigma(\varphi)) .
$$

This leads us to one of our main results.

7.18. Theorem. Let $S$ be a 0-left-cancellative semigroup admitting least common multiples. Then, for every open, maximal string $\sigma$ over $S$, one has that $\varphi_{\sigma}$ is an ultra-character.

The previous result raises the question as to whether $\sigma_{\varphi}$ is a maximal string for every ultra-character $\varphi$, but this is not true in general. Consider for example the unital semigroup

$$
S=\{1, a, a a, 0\},
$$

in which $a^{3}=0$. The $\theta$-constructible subsets of $S$ are precisely

\begin{tabular}{|c|c|c|}
\hline$E_{1}^{\theta}=F_{1}^{\theta}=\{1, a, a a\}$ & & \\
\hline$F_{a}^{\theta}=\{1, a\}$ & $E_{a}^{\theta}=a F_{a}^{\theta}=\{a, a a\}$ & \\
\hline$F_{a a}^{\theta}=\{1\}$ & $a F_{a a}^{\theta}=\{a\}$ & $E_{a a}^{\theta}=a a F_{a a}^{\theta}=\{a a\}$ \\
\hline \multicolumn{3}{|c|}{ List of $\theta$-constructible sets } \\
\hline
\end{tabular}

and there are three strings over $S$, namely

$$
\begin{array}{|l|l|l|}
\hline \delta_{1}=\{1\} & \delta_{a}=\{1, a\} & \delta_{a a}=\{1, a, a a\} \\
\hline
\end{array}
$$

Since the correspondence $s \mapsto \delta_{s}$ is a bijection from $S^{\prime}$ to $S^{\star}$, we see that $\theta^{\star}$ is isomorphic to $\theta$, and in particular the $\theta^{\star}$-constructible subsets of $S^{\star}$, listed below, mirror the $\theta$-constructible ones.

\begin{tabular}{|c|c|c|}
\hline$E_{1}^{\star}=F_{1}^{\star}=\left\{\delta_{1}, \delta_{a}, \delta_{a a}\right\}$ & & \\
\hline$F_{a}^{\star}=\left\{\delta_{1}, \delta_{a}\right\}$ & $E_{a}^{\star}=a F_{a}^{\star}=\left\{\delta_{a}, \delta_{a a}\right\}$ & \\
\hline$F_{a a}^{\theta}=\left\{\delta_{1}\right\}$ & $a F_{a a}^{\star}=\left\{\delta_{a}\right\}$ & $E_{a a}^{\theta}=a a F_{a a}^{\star}=\left\{\delta_{a a}\right\}$ \\
\hline \multicolumn{3}{|c}{ List of $\theta^{\star}$-constructible sets }
\end{tabular}

Observe that the string $\sigma:=\delta_{a}=\{1, a\}$ is a proper subset of the string $\{1, a, a a\}$, and hence $\sigma$ is not maximal. But yet notice that $\varphi_{\sigma}$ is an ultra-character, since $\left\{\delta_{a}\right\}$ is a minimal ${ }^{4}$ member of $\mathcal{P}\left(S^{\star}, \theta^{\star}\right)$. We thus get an example of

"A string $\sigma$ which is not maximal but such that $\varphi_{\sigma}$ is an ultra-character."

On the other hand, since $\sigma=\sigma_{\varphi_{\sigma}}$, this also provides an example of

"An ultra-character $\varphi$ such that $\sigma_{\varphi}$ is not maximal."

This suggests the need to single out the strings which give rise to ultra-characters:

7.19. Definition. We will say that a string $\sigma$ is quasi-maximal whenever $\varphi_{\sigma}$ is an ultra-character. The set of all quasi-maximal strings will be denoted by $S^{\propto}$.

Adopting this terminology, the conclusion of (7.18) states that every open, maximal string is quasimaximal.

\footnotetext{
4 Whenever $e_{0}$ is a nonzero minimal element of a semilattice $E$, the character $\varphi(e)=\left[e_{0} \leq e\right]$ is an ultra-character.
} 
7.20. Theorem. Let $S$ be a 0-left-cancellative semigroup admitting least common multiples. Then, every open ultra-character on $\mathfrak{E}(S)$ is of the form $\varphi_{\sigma}$ for some open, quasi-maximal string $\sigma$.

The importance of quasi-maximal strings evidenced by the last result begs for a better understanding of such strings. While we are unable to provide a complete characterization, we can at least exhibit some further examples beyond the maximal ones.

To explain what we mean, recalll from (6.9.i) that a string $\sigma$ belongs to some $F_{\Lambda}^{\star}$ if and only if $\sigma$ is contained in $F_{\Lambda}^{\theta}$. It is therefore possible that $\sigma$ is maximal among all strings contained in $F_{\Lambda}^{\theta}$, and still not be a maximal string. An example is the string $\{1, a\}$ mentioned above, which is maximal within $F_{a}^{\theta}$, but not maximal in the strict sense of the word.

7.21. Proposition. Let $\Lambda$ be a nonempty finite subset of $S$ and suppose that $\sigma$ is an open string such that $\sigma \subseteq F_{\Lambda}^{\theta}$. Suppose moreover that $\sigma$ is maximal among the strings contained in $F_{\Lambda}^{\theta}$, in the sense that for every string $\mu$, one has that

$$
\sigma \subseteq \mu \subseteq F_{\Lambda}^{\theta} \Rightarrow \sigma=\mu .
$$

Then $\varphi_{\sigma}$ is an ultra-character, and hence $\sigma$ is a quasi-maximal string.

\section{Ground characters.}

In the last section we were able to fruitfully study open characters using strings, culminating with Theorem (7.20), stating that every open ultra-character is given in terms of a string. However nothing of interest was said about an ultra-character when it is not open. The main purpose of this section is thus to obtain some useful information about non-open ultra-characters. The main result in this direction is Theorem (8.11), below.

- Throughout this section we fix a 0-left-cancellative semigroup $S$ admitting least common multiples. For each $s$ in $S$ let

$$
\hat{F}_{s}=\left\{\varphi \in \widehat{\mathfrak{E}}(S): \varphi\left(F_{s}^{\theta}\right)=1\right\}, \quad \text { and } \quad \hat{E}_{s}=\left\{\varphi \in \widehat{\mathfrak{E}}(S): \varphi\left(E_{s}^{\theta}\right)=1\right\},
$$

and for every $\varphi$ in $\hat{F}_{s}$, consider the character $\hat{\theta}_{s}(\varphi)$ given by

$$
\hat{\theta}_{s}(\varphi)(X)=\varphi\left(\theta_{s}^{-1}\left(E_{s}^{\theta} \cap X\right)\right), \quad \forall X \in \mathfrak{E}(S) .
$$

Observing that

$$
\hat{\theta}_{s}(\varphi)\left(E_{s}^{\theta}\right)=\varphi\left(\theta_{s}^{-1}\left(E_{s}^{\theta}\right)\right)=\varphi\left(F_{s}^{\theta}\right)=1,
$$

we see that $\hat{\theta}_{s}(\varphi)$ is indeed a (nonzero) character, and that $\hat{\theta}_{s}(\varphi)$ belongs to $\hat{E}_{s}$. As a consequence we get a map

$$
\hat{\theta}_{s}: \hat{F}_{s} \rightarrow \hat{E}_{s}
$$

which is easily seen to be bijective, with inverse given by

$$
\hat{\theta}_{s}^{-1}(\varphi)(X)=\varphi\left(\theta_{s}\left(F_{s}^{\theta} \cap X\right)\right), \quad \forall \varphi \in \hat{E}_{s}, \quad \forall X \in \mathfrak{E}(S) .
$$

We may then see each $\hat{\theta}_{s}$ as an element of $\mathcal{I}(\widehat{\mathfrak{E}}(S))$, and it is not hard to see that the correspondence

$$
\hat{\theta}: s \in S \mapsto \hat{\theta}_{s} \in \mathcal{I}(\widehat{\mathfrak{E}}(S))
$$

is a representation of $S$ on $\widehat{\mathfrak{E}}(S)$.

All of this may also be deduced from the fact that any inverse semigroup, such as $\mathfrak{H}(S)$, admits a canonical representation on the spectrum of its idempotent semilattice (see [7: Section 10]), and that $\hat{\theta}$ may be obtained as the composition

$$
S \stackrel{\theta}{\longrightarrow} \mathfrak{H}(S) \longrightarrow \mathcal{I}(\widehat{\mathfrak{E}}(S)),
$$

where the arrow in the right-hand-side is the canonical representation mentioned above. 
8.2. Definition. We shall refer to $\hat{\theta}$ as the dual representation of $S$.

The following technical result gives some useful information regarding the relationship between the dual representation and the representation $\rho$ of $\mathfrak{H}(S)$ described in (6.15).

8.3. Lemma. Given $s$ in $S$, and $\sigma$ in $S_{\sharp}^{\star}$, one has that

(i) $\varphi_{\sigma} \in \hat{F}_{s} \Leftrightarrow \sigma \in F_{s}^{\star}$,

(ii) if the equivalent conditions in (i) are satisfied, then $\hat{\theta}_{s}\left(\varphi_{\sigma}\right)=\varphi_{\theta_{s}^{\star}(\sigma)}$,

(iii) $\varphi_{\sigma} \in \hat{E}_{s} \Leftrightarrow \sigma \in E_{s}^{\star}$,

(iv) if the equivalent conditions in (iii) are satisfied, then $\hat{\theta}_{s}^{-1}\left(\varphi_{\sigma}\right)=\varphi_{\theta_{s}^{\star-1}(\sigma)}$.

Considering the representation $\theta^{\star}$ of $S$ on $S^{\star}$, observe that $S_{\sharp}^{\star}$ is an invariant subset of $S^{\star}$, and it is easy to see that it is also invariant under the representation $\rho$ of $\mathfrak{H}(S)$ described in (6.15). Together with the dual representation of $\mathfrak{H}(S)$ on $\widehat{\mathfrak{E}}(S)$ mentioned above, we thus have two natural representations of $\mathfrak{H}(S)$, which are closeely related, as the following immediate consequence of the above result asserts:

8.4. Proposition. The mapping

$$
\Phi: S_{\sharp}^{\star} \rightarrow \widehat{\mathfrak{E}}(S)
$$

of (7.7) is covariant relative to the natural representations of $\mathfrak{H}(S)$ referred to above.

The fact that the correspondence between strings and characters is not a perfect one (see e.g. (7.13) and (7.17)) is partly responsible for the fact that expressing the covariance properties of the map $\Sigma$ of (7.12) cannot be done in the same straightforward way as we did for $\Phi$ in (8.4). Nevertheless, there are some things we may say in this respect.

Let us first treat the question of covariance regarding $\hat{\theta}_{s}^{-1}(\varphi)$. Of course, for this to be a well defined character we need $\varphi$ to be in $\hat{E}_{s}$, meaning that $\varphi\left(E_{s}^{\theta}\right)=1$, which is also equivalent to saying that $s \in \sigma_{\varphi}$. In particular characters with empty strings are immediately ruled out.

8.5. Lemma. For every $s$ in $S$, and every character $\varphi$ in $\hat{E}_{s}$, one has that

$$
\sigma_{\hat{\theta}_{s}^{-1}(\varphi)}=\left\{p \in S: s p \in \sigma_{\varphi}\right\} .
$$

The set appearing in the right hand side of the equation displayed in (8.5) is precisely the same set mentioned in definition (6.6.ii) of $s^{-1} * \sigma_{\varphi}$, except that this notation is reserved for the situation in which the intersection of $\sigma$ with $s S$ is nonempty, which precisely means that $s \in \circ^{\circ}$.

8.6. Proposition. Pick $s$ in $S$ and let $\varphi$ be any character in $\hat{E}_{s}$. Then $s \in \sigma_{\varphi}$, and moreover

(i) if $s$ is in $\stackrel{\circ}{\varphi}_{\varphi}$, then $\sigma_{\varphi} \in E_{s}^{\star}$, and $\sigma_{\hat{\theta}_{s}^{-1}(\varphi)}=\theta_{s}^{\star-1}\left(\sigma_{\varphi}\right)$,

(ii) if $s$ is not in $\stackrel{\circ}{\varphi}_{\varphi}$, then $\sigma_{\hat{\theta}_{s}^{-1}(\varphi)}=\emptyset$.

Regarding the behavior of strings associated to characters of the form $\hat{\theta}_{s}(\varphi)$, we have:

8.7. Lemma. For every $s$ in $S$, and every character $\varphi$ in $\hat{F}_{s}$, one has that $\hat{\theta}_{s}(\varphi)$ belongs to $\widehat{\mathfrak{E}}_{1}(S)$ (and hence (7.10) implies that $\sigma_{\hat{\theta}_{s}(\varphi)}$ is a string), and moreover

(i) if $\sigma_{\varphi}$ is nonempty, then $\sigma_{\varphi} \in F_{s}^{\star}$, and $\sigma_{\hat{\theta}_{s}(\varphi)}=\theta_{s}^{\star}\left(\sigma_{\varphi}\right)$,

(ii) if $\sigma_{\varphi}$ is empty, then $\sigma_{\hat{\theta}_{s}(\varphi)}=\delta_{s}$.

We may interpret the above result, and more specifically the identity

$$
\sigma_{\hat{\theta}_{s}(\varphi)}=\theta_{s}^{\star}\left(\sigma_{\varphi}\right)
$$

as saying that the correspondence $\varphi \mapsto \sigma_{\varphi}$ is covariant with respect to the actions $\hat{\theta}$ and $\theta^{\star}$, on $\widehat{\mathfrak{E}}(S)$ and $S^{\star}$, respectively, except that the term " $\sigma_{\varphi}$ " appearing is the right-hand-side above is not a well defined string since it may be empty, even though the left-hand-side is always well defined. In the problematic case of an empty string, (8.7.ii) then gives the undefined right-hand-side the default value of $\delta_{s}$. 
8.8. Definition. A character $\varphi$ in $\widehat{\mathfrak{E}}(S)$ will be called a ground character if $\sigma_{\varphi}$ is empty.

By (7.10), the ground characters are precisely the members of $\widehat{\mathfrak{E}}(S) \backslash \widehat{\mathfrak{E}}_{1}(S)$.

Besides the ground characters, a character $\varphi$ may fail to be open because $\sigma_{\varphi}$, while being a bona fide string, is not an open string. In this case we have that $\sigma_{\varphi}=\delta_{s}$, for some $s$ in $S$ such that $s \notin s S$.

8.9. Proposition. Let $\varphi$ be a character such that $\sigma_{\varphi}=\delta_{s}$, where $s$ is such that $s \notin s S$. Then $\varphi \in \hat{E}_{s}$, and $\hat{\theta}_{s}^{-1}(\varphi)$ is a ground character.

We may now give a precise characterization of non-open characters in terms of the ground characters:

8.10. Proposition. Denote by $\widehat{\mathfrak{E}}_{\mathrm{op}}(S)$ the set of all open characters on $\mathfrak{E}(S)$. Then

$$
\widehat{\mathfrak{E}}(S) \backslash \widehat{\mathfrak{E}}_{\mathrm{op}}(S)=\left\{\hat{\theta}_{u}(\varphi): u \in \tilde{S}, \varphi \text { is a ground character in } \hat{F}_{u}\right\} .
$$

Moreover for each $\psi$ in the above set, there is a unique pair $(u, \varphi)$, with $u$ in $\tilde{S}$, and $\varphi$ a ground character, such that $\psi=\hat{\theta}_{u}(\varphi)$.

We may now combine several of our earlier results to give a description of all ultra-characters on $\mathfrak{E}(S)$.

8.11. Theorem. Let $S$ be a 0-left-cancellative semigroup admitting least common multiples. Denote by $\widehat{\mathfrak{E}}_{\infty}(S)$ the set of all ultra-characters on $\mathfrak{E}(S)$, and by

$$
\widehat{\mathfrak{E}}_{\infty}^{\mathrm{op}}(S)=\widehat{\mathfrak{E}}_{\mathrm{op}}(S) \cap \widehat{\mathfrak{E}}_{\infty}(S),
$$

namely the subset formed by all open ultra-characters. Then

(i) $\widehat{\mathfrak{E}}_{\infty}^{\text {op }}(S) \quad=\left\{\varphi_{\sigma}: \sigma\right.$ is an open, quasi-maximal string in $\left.S\right\}$, and

(ii) $\widehat{\mathfrak{E}}_{\infty}(S) \backslash \widehat{\mathfrak{E}}_{\infty}^{\mathrm{op}}(S)=\left\{\hat{\theta}_{u}(\varphi): u \in \tilde{S}, \varphi\right.$ is a ground, ultra-character in $\left.\hat{F}_{u}\right\}$.

The upshot is that in order to understand all ultra-characters on $\mathfrak{E}(S)$, it only remains to describe the ground ultra-characters.

\section{Subshift semigroups.}

By a subshift on a finite alphabet $\Sigma$ one means a subset $\mathcal{X} \subseteq \Sigma^{\mathbb{N}}$, which is closed relative to the product topology, and invariant under the left shift map

$$
x_{1} x_{2} x_{3} \ldots \mapsto x_{2} x_{3} x_{4} \ldots
$$

- Throughout this chapter we will let $\mathcal{X}$ be a fixed subshift.

The language of $\mathcal{X}$ is the set $L$ formed by all finite words appearing as a block in some infinite word belonging to $\mathcal{X}$. We will not allow the empty word in $L$, as sometimes done in connection with subshifts, so all of our words have strictly positive length.

In the present section we will be concerned with the semigroup

$$
S_{\mathcal{X}}=L \cup\{0\},
$$

equipped with the multiplication operation given by

$$
\mu \cdot \nu=\left\{\begin{array}{cl}
\mu \nu, & \text { if } \mu, \nu \neq 0, \text { and } \mu \nu \in L, \\
0, & \text { otherwise }
\end{array}\right.
$$

where $\mu \nu$ stands for the concatenation of $\mu$ and $\nu$.

Given $\mu$ and $\nu$ in $S_{\mathcal{X}}$, with $\nu$ nonzero, notice that $\mu \mid \nu$ if and only if $\mu$ is a prefix of $\nu$. Given that divisibility is also well defined for the unitized semigroup $\tilde{S}_{\mathcal{X}}$, and that 1 divides any $\mu \in S_{\mathcal{X}}$, we will also say that 1 is a prefix of $\mu$.

Some of the special properties of $S_{\mathcal{X}}$ of easy verification are listed below: 


\subsection{Proposition.}

(i) $S_{\mathcal{X}}$ is 0-left-cancellative and 0-right-cancellative,

(ii) $S_{\mathcal{X}}$ admits least common multiples,

(iii) $S_{\mathcal{X}}$ has no idempotent elements other than 0.

A further special property of $S_{\mathcal{X}}$ is a very strong uniqueness of the normal form for elements in $\mathfrak{H}\left(S_{\mathcal{X}}\right)$ :

9.3. Proposition. For $i=1,2$, let $\Lambda_{i}$ be a finite subset of $\tilde{S}_{\mathcal{X}}$ intersecting $S_{\mathcal{X}}$ non-trivially, and let $u_{i}, v_{i} \in \Lambda_{i}$ be such that

$$
\theta_{u_{1}} f_{\Lambda_{1}} \theta_{v_{1}}^{-1}=\theta_{u_{2}} f_{\Lambda_{2}} \theta_{v_{2}}^{-1} \neq 0 .
$$

Then $u_{1}=u_{2}, v_{1}=v_{2}$, and $F_{\Lambda_{1}}=F_{\Lambda_{2}}$.

Given the importance of strings, let us give an explicit description of these in the present context.

9.4. Proposition. Given a (finite or infinite) word

$$
\omega=\omega_{1} \omega_{2} \omega_{3} \ldots
$$

on the alphabet $\Sigma$, assume $\omega$ to be admissible (meaning that $\omega$ belongs to $L$, if finite, or to $\mathcal{X}$, if infinite) and consider the set $\delta_{\omega}$ formed by all prefixes of $\omega$ having positive length, namely

$$
\delta_{\omega}=\left\{\omega_{1}, \omega_{1} \omega_{2}, \omega_{1} \omega_{2} \omega_{3}, \ldots\right\} .
$$

Then:

(i) $\delta_{\omega}$ is a string,

(ii) $\delta_{\omega}$ is an open string if and only if $\omega$ is an infinite word,

(iii) $\delta_{\omega}$ is a maximal string if and only if $\omega$ is an infinite word,

(iv) for any string $\sigma$ in $S_{\mathcal{X}}$, there exists a unique admissible word $\omega$ such that $\sigma=\delta_{\omega}$.

Strings consist in one of our best instruments to provide characters on $\mathfrak{E}\left(S_{\mathcal{X}}\right)$. Now that we have a concrete description of strings in terms of admissible words, let us give an equally concrete description of the characters induced by strings.

9.5. Proposition. Let $\omega$ be a given infinite admissible word, and let $X$ be any $\theta$-constructible set, written in normal form, namely $X=u F_{\Lambda}^{\theta}$, where $\Lambda$ is a finite subset of $\tilde{S}_{\mathcal{X}}$, intersecting $S_{\mathcal{X}}$ nontrivially, and $u \in \Lambda$. Regarding the string $\delta_{\omega}$, and the associated character $\varphi_{\delta_{\omega}}$, the following are equivalent:

(i) $\varphi_{\delta_{\omega}}(X)=1$,

(ii) $u$ is a prefix of $\omega$, and upon writing $\omega=u \eta$, for some infinite word $\eta$, one has that $t \eta$ is admissible (i.e. lies in $\mathcal{X}$ ), for every $t$ in $\Lambda$.

In case the set $X$ of the above result coincides with $F_{\mu}^{\theta}$, for some $\mu$ in $L$, we get the following simplification:

9.6. Proposition. Let $\omega$ be an infinite admissible word, and let $\mu \in L$. Then

$$
\varphi_{\delta_{\omega}}\left(F_{\mu}^{\theta}\right)=1 \quad \Leftrightarrow \quad \mu \omega \in \mathcal{X} .
$$

Let us now study a situation in which infinite words provide all ultra-characters.

9.7. Proposition. The following are equivalent:

(i) for every finite subset $\Lambda \subseteq \tilde{S}_{\mathcal{X}}$, such that $\Lambda \cap S_{\mathcal{X}} \neq \emptyset$, one has that $F_{\Lambda}^{\theta}$ is either empty or infinite,

(ii) every nonempty constructible set is infinite,

(iii) $\left\{E_{a}^{\theta}: a \in \Sigma\right\}$ is a cover for $\mathfrak{E}\left(S_{\mathcal{X}}\right)$,

(iv) $\mathfrak{E}\left(S_{\mathcal{X}}\right)$ admits no ground ultra-characters,

(v) for every ultra-character $\varphi$ on $\mathfrak{E}\left(S_{\mathcal{X}}\right)$, there exists an infinite admissible word $\omega$, such that $\varphi=\varphi_{\delta_{\omega}}$. 
Let us conclude this section with an example to show that nonempty finite constructible sets may indeed exist and hence the equivalent conditions of (9.7) do not always hold. Consider the alphabet $\Sigma=\{a, b, c\}$ and let $\mathcal{X}$ be the subshift on $\Sigma$ consisting of all infinite words $\omega$ such that, in any block of $\omega$ of length three, there are no repeated letters. Alternatively, a set of forbidden words defining $\mathcal{X}$ is the set of all words of length three with some repetition.

It is then easy to see that the language $L$ of $\mathcal{X}$ is formed by all finite words on $\Sigma$ with the same restriction on blocks of length three described above.

Notice that $c \in F_{\{a, b\}}$, because both $a c$ and $b c$ are in $L$. However there is no element in $F_{\{a, b\}}$ other than $c$, because it is evident that neither $a$ nor $b$ lie in $F_{\{a, b\}}$, and for any $x$ in $\Sigma$, either $a c x$ or $b c x$ will involve a repetition. So voilà the finite constructible set:

$$
F_{\{a, b\}}=\{c\} .
$$

\section{C*-algebras associated to subshifts.}

In this final section we will briefly discuss applications of the theory so far described to various $\mathrm{C}^{*}$-algebras associated to subshifts that have been studied starting with Matsumoto's original work [11].

Given a subshift $\mathcal{X}$, as in the previous section, we will consider the 0-left-cancellative semigroup $S_{\mathcal{X}}$, as well as its inverse hull $\mathfrak{H}\left(S_{\mathcal{X}}\right)$. We may then consider several general constructions of $\mathrm{C}^{*}$-algebras from inverse semigroups, and our goal is to argue that many of these, once applied to $\mathfrak{H}\left(S_{\mathcal{X}}\right)$, produce all of the $\mathrm{C}^{*}$-algberas studied in the literature in connection with subshifts.

The constructions we have in mind share a common pattern in the following sense. Given an inverse semigroup $\mathcal{S}$ with zero, consider the standard action of $\mathcal{S}$ on $\widehat{E}(\mathcal{S})$, namely the dual of the idempotent semilattice of $\mathcal{S}$. We may then build the groupoid $\mathcal{G}_{\mathcal{S}}$ formed by all germs for this action. This groupoid is sometimes referred to via the suggestive notation

$$
\mathcal{S} \ltimes \widehat{E}(\mathcal{S}) .
$$

The $\mathrm{C}^{*}$-algebra of $\mathcal{G}_{\mathcal{S}}$ is well known to be a quotient ${ }^{5}$ of Paterson's universal $\mathrm{C}^{*}$-algebra for $\mathcal{S}$.

Whenever $Y$ is a closed invariant subset of $\widehat{E}(\mathcal{S})$, we may restrict the action of $\mathcal{S}$ to $Y$, and consider the corresponding groupoid of germs $\mathcal{S} \ltimes Y$, which may also be seen as the reduction of $\mathcal{G}_{\mathcal{S}}$ to $Y$.

Our point is that several $\mathrm{C}^{*}$-algebras studied in the literature in connection to the subshift $\mathcal{X}$ are actually groupoid $\mathrm{C}^{*}$-algebras of the form

$$
C^{*}\left(\mathfrak{H}\left(S_{\mathcal{X}}\right) \ltimes Y\right),
$$

where $Y$ is a closed subset of $\widehat{\mathfrak{E}}\left(S_{\mathcal{X}}\right)$, invariant under the standard action of $\mathfrak{H}\left(S_{\mathcal{X}}\right)$.

In order to describe the first relevant alternative for $Y$, let $S$ be any 0-left-cancellative semigroup and consider the representation $\iota$ of $\mathfrak{E}(S)$ on $\mathcal{P}\left(S^{\prime}\right)$ given by the inclusion of the former in the latter. We will say that a character $\varphi$ of $\mathfrak{E}(S)$ is essentially tight (relative to the above representation $\iota$ ) provided one has that

$$
\varphi(X)=\bigvee_{i=1}^{n} \varphi\left(Y_{i}\right)
$$

whenever $X, Y_{1}, \ldots, Y_{n}$ are in $\mathfrak{E}(S)$, and the symmetric difference

$$
X \Delta\left(\bigcup_{i=1}^{n} Y_{i}\right)
$$

is finite. The set of all essentially tight characters of $\mathfrak{E}(S)$ will be denoted by $\widehat{\mathfrak{E}}_{\text {ess }}(S)$, and it may be shown that $\widehat{\mathfrak{E}}_{\text {ess }}(S)$ is a closed invariant subset of $\widehat{\mathfrak{E}}(S)$.

\footnotetext{
5 Modulo the relation that identifies the zero of $\mathcal{S}$ with the zero of the corresponding $\mathrm{C}^{*}$-algebra.
} 
The second relevant alternative for $Y$ is based on the set $\widehat{\mathfrak{E}}_{\max }(S)$ defined by

$$
\widehat{\mathfrak{E}}_{\max }(S)=\left\{\varphi_{\sigma}: \sigma \text { is a maximal string }\right\} .
$$

In general $\widehat{\mathfrak{E}}_{\text {max }}(S)$ is not invariant under the action of $\mathfrak{H}(S)$, but when $\mathcal{S}=S_{\mathcal{X}}$ for some subshift $\mathcal{X}$, invariance is guaranteed. One may then take $Y$ to be the closure of $\widehat{\mathfrak{E}}_{\max }\left(S_{\mathcal{X}}\right)$.

The tight spectrum $\widehat{\mathfrak{E}}_{\text {tight }}\left(S_{\mathcal{X}}\right)$ is a further alternative, and in some sense it is the most natural one given that many $\mathrm{C}^{*}$-algebras associated to inverse semigroups turn out to be the groupoid $\mathrm{C}^{*}$-algebra for the reduction of Paterson's universal groupoid to the tight spectrum of the idempotent semilattice.

These subsets are related to each other as follows

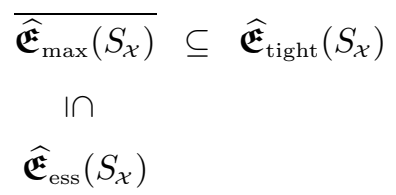

As already observed, all of these are subsets of $\mathfrak{E}\left(S_{\mathcal{X}}\right)$ which are closed and invariant under the action of $\mathfrak{H}\left(S_{\mathcal{X}}\right)$, so each gives rise to a reduced subgroupoid, which we will correspondingly denote by $\mathcal{G}_{\text {max }}, \mathcal{G}_{\text {tight }}$ and $\mathcal{G}_{\text {ess }}$.

10.2. Theorem. Given any subshift $\mathcal{X}$, one has that

(i) $C^{*}\left(\mathcal{G}_{\text {ess }}\right)$ is isomorphic to Matsumoto's $C^{*}$-algebra introduced in [11].

(ii) $C^{*}\left(\mathcal{G}_{\max }\right)$ is isomorphic to Carlsen-Matsumoto's $C^{*}$-algebra introduced in [1: Definition 2.1].

There are many situations in which the inclusions in (10.1) reduce to equality, but examples may be given to show these are, in general, proper inclusions. The fact that ${\widehat{\mathfrak{E}_{\text {max }}\left(S_{\mathcal{X}}\right)}}_{\text {and }} \widehat{\mathfrak{E}}_{\text {ess }}\left(S_{\mathcal{X}}\right)$ may differ is related to the fact that Matsumoto's C*-algebra may be non-isomorphic to the Carlsen-Matsumoto one, but it may be shown that under condition $(*)^{6}$ of $[\mathbf{1}]$, one has that $\overline{\widehat{\mathfrak{E}}_{\text {max }}\left(S_{\mathcal{X}}\right)}=\widehat{\mathfrak{E}}_{\mathrm{ess}}\left(S_{\mathcal{X}}\right)$, whence isomorphism holds.

\section{REFERENCES}

[1] T. M. Carlsen and K. Matsumoto, "Some remarks on the C*-algebras associated with subshifts", Math. Scand., 95 (2004), $145-160$.

[2] A. Cherubini and M. Petrich, "The Inverse Hull of Right Cancellative Semigroups", J. Algebra, 111 (1987), 74-113.

[3] A. H. Clifford and G. B. Preston, "The algebraic theory of semigroups. Vol. I", Mathematical Surveys, No. 7. American Mathematical Society, Providence, R.I., 1961.

[4] L. A. Coburn, "The C*-algebra generated by an isometry I", Bull. Amer. Math. Soc., 73 (1967), 722-726.

[5] L. A. Coburn, "The C*-algebra generated by an isometry II", Trans. Amer. Math. Soc., 137 (1969), $211-217$.

[6] M. Dokuchaev and R. Exel, "Partial actions and subshifts", J. Funct. Analysis, 272 (2017), 5038-5106.

[7] R. Exel, "Inverse semigroups and combinatorial C*-algebras", Bull. Braz. Math. Soc. (N.S.), 39 (2008), $191-313$.

[8] R. Exel and M. Laca, "Cuntz-Krieger algebras for infinite matrices", J. reine angew. Math., 512 (1999), $119-172$.

[9] R. Exel and E. Pardo, "The tight groupoid of an inverse semigroup", Semigroup Forum, 92 (2016), 274-303.

[10] X. Li, "Semigroup C*-algebras and amenability of semigroups", J. Funct. Anal., 262 (2012), 4302-4340.

[11] K. Matsumoto, "On C*-algebras associated with subshifts", Internat. J. Math., 8 (1997), 357-374.

[12] M. Morse and G. A. Hedlund, "Unending chess, symbolic dynamics and a problem in semigroups", Duke Math. J., 11 (1944), 1-7..

[13] W. D. Munn, "Brandt congruences on inverse semigroups", Proc. London Math. Soc., (3) 14 (1964), 154-164..

[14] G. J. Murphy, "Ordered groups and Toeplitz algebras", J. Operator Theory, 18 (1987), 303-326.

[15] G. J. Murphy, "Ordered groups and crossed products of C*-algebras", Pacific J. Math., 2 (1991), 319-349.

[16] G. J. Murphy, "Crossed products of C*-algebras by semigroups of automorphisms", Proc. London Math. Soc., 3 (1994), 423-448.

[17] A. Nica, "C*-algebras generated by isometries and Wiener-Hopf operators", J. Operator Theory, 27 (1992), 17-52.

[18] A. L. T. Paterson, "Groupoids, inverse semigroups, and their operator algebras", Birkhäuser, 1999.

6 The reader should be warned that the description of condition $(*)$ in $[\mathbf{1}]$ is incorrect and must be amended by requiring that the sequence $\left\{u_{i}\right\}_{i}$, mentioned there, have an infinite range. 